\title{
EMERGÊNCIA TEMÁTICA DA INOVAÇÃO SUSTENTÁVEL: UMA ANÁLISE DA PRODUÇÃO CIENTIIFICA ATRAVÉS DA BASE WEB OF SCIEN̉CE
}

\author{
EMERGENCY OF THE SUSTAINABLE INNOVATION \\ THEMATIC: AN ANALYSIS OF SCIENTIFIC PRODUCTION \\ THROUGH WEB OF SCIENCE BASE
}

Recebido 22/09/2011

Aceito 15/11/2011

Jordana Marques Kneipp ${ }^{1}$, Luciana Aparecida Barbieri da Rosa² ${ }^{2}$, Roberto Schoproni Bichueti $^{3}$, Lúcia Rejane da Rosa Gama Madruga ${ }^{4}$ e Vitor Francisco Schuch Júnior ${ }^{5}$

\section{RESUMO}

A inovação como fator de competitividade necessita considerar seus efeitos sobre a sociedade e o meio ambiente, de modo a abranger aspectos do ambiente organizacional. Nesse sentido, estudos vêm sendo desenvolvidos com o intuito de englobar duas temáticas emergentes: inovação e sustentabilidade. A fim de ampliar o conhecimento referente à produção científica relacionada à inovação sustentável, este estudo teve como objetivo analisar as características das publicações sobre Sustainable Innovation (Inovação Sustentável), na base de dados Web of Science, no período de 2000 a 2010, e identificar quais tópicos em administração estudados junto a este tema estão sendo mais pesquisados e quais são mais relevantes (hot topics). O trabalho caracteriza-se como exploratório e descritivo, pois se trata de um estudo de natureza bibliométrica, que busca o aprofundamento da análise da produção acadêmica sobre inovação sustentável. Como principais resultados das 1.022 publicações analisadas, constatou-se aumento gradual da produção científica, em especial nas seguintes áreas temáticas: Gestão (Management), Ciências Ambientais (Environmental Sciences) e Negócio (Business). Evidenciou-se como hot topcis as combinações do tópico inovação sustentável com: Development (Desenvolvimento), Management (Gestão) e Technology (Tecnologia).

Palavras-chave: Inovação. Sustentabilidade. Bibliometria.

\footnotetext{
1 Mestranda do Programa de Pós-Graduação em Administração da Universidade Federal de Santa Maria UFSM). E-mail: jordanakneipp@yahoo.com.br

2 Mestranda do Programa de Pós-Graduação em Administração da UFSM.

E-mail: lucianaaparecidabarbieri@yahoo.com.br

3 Mestrando no Programa de Pós-Graduação em Administração da UFSM. E-mail: robertobichueti@hotmail.com

4 Professora do Departamento de Ciências Administrativas da UFSM. E-mail: lucia.rejane@hotmail.com

5 Professor Visitante do Departamento de Ciências Administrativas da UFSM. E-mail: vfschuch@gmail.com
} 


\section{ABSTRACT}

Innovation as a competitive factor needs to consider its effects on society and the environment, to cover aspects of the organization environment. In this way, several studies have been developed in order to include two emerging themes: innovation and sustainability. In order to increase the knowledge on the scientific production related to sustainable innovation, this study aimed to analyze the characteristics of publications on sustainable innovation, on Web of Science database, in period of 2000 to 2010, and indentify witch Administration topics studied with this theme are most researched and witch are most relevant (hot topics). The job characterized as exploratory and descriptive, because is a bibliometric study of nature, that seeks to deepen de analyzes the scientific/academic production about sustainable innovation. In the main results of 1.022 publications analyzed, there was the gradual increase in scientific production, particularly in these areas: Management, Environmental Sciences and Business. As hot topics, the first highlights was the combinations of the sustainable innovation with: Development, Management as Technology.

Keywords: Innovation. Sustainable. Bibliometic study.

\section{INTRODUÇÃO}

A obtenção de vantagem competitiva pelas organizações a partir do desenvolvimento de práticas inovadoras é cada vez mais recorrente nas suas estratégias de negócio. Dessa forma, torna-se primordial o desenvolvimento de novas práticas de gestão que impulsionem melhorias em produtos e processos, transformando-se em um fator de competitividade. Bessant e Tidd (2009) vão além ao afirmar que a inovação é uma questão de sobrevivência para as empresas, devido à alta competitividade presente no ambiente empresarial.

A inovação, segundo Schumpeter (1961), é conceituada como um processo marcado pela descontinuidade do que já está estabelecido e concebida por meio da introdução de um novo bem ou nova qualidade de um bem, de um novo método de produção, da abertura de um novo mercado, da conquista de uma nova fonte de matéria-prima ou, até mesmo, da criação de uma nova forma de organização.

Na visão de Daroit e Nascimento (2004), a inovação está comumente associada ao enfoque econômico, sendo tratada apenas como forma de obtenção de lucros extras pelas empresas, por meio de vantagens competitivas decorrentes da produção de novos produtos ou processos que agregam valor para o cliente. No entanto, estes autores enfatizam que é preciso uma maior compreensão por parte das organizações sobre o papel da inovação com relação a seus efeitos sobre a sociedade e o meio ambiente.

Considerar os efeitos sobre o ambiente corrobora o novo enfoque no âmbito organizacional, voltado para o desenvolvimento sustentável, que busca unir o bem-estar econômico, a equidade social e a proteção ao meio ambiente.

As organizações sustentáveis são aquelas que desenvolvem suas práticas e estratégias gerenciais a fim de serem economicamente viáveis, mantendo-se competitivas no mercado, produzindo de maneira a não agredir o meio ambiente e contribuindo para o desenvolvimento social da região e do país onde atuam (ALMEIDA, 2002; LEAL, 2009).

A inovação direcionada para o desenvolvimento sustentável requer tanto a incorporação das restrições oriundas das pressões sociais e ambientais como a inclusão de uma visão que considere também as futuras gerações, ou seja, que tenha uma perspectiva de longo prazo (HALL; VREDENBURG, 2003). A fim de ampliar o conhecimento referente à produção científica relacionada à inovação sustentável, este estudo teve como objetivo analisar as características 
das publicações sobre Sustainable Innovation (Inovação Sustentável), na base de dados Web of Science, no período de 2000 a 2010, e identificar quais tópicos em administração estudados junto a este tema estão sendo mais pesquisados e quais são mais relevantes (hot topics).

Este estudo está estruturado da seguinte maneira: inicialmente, será apresentada uma contextualização sobre a emergência da inovação sustentável. Em seguida, evidencia-se o método utilizado para o desenvolvimento deste estudo, após apresenta-se a análise e discussão dos resultados encontrados e, por fim, as considerações finais.

\section{INOVAÇÃO SUSTENTÁVEL: UMA TEMÁTICA EMERGENTE}

A inovação consiste em uma temática bastante ampla, já que pode estar relacionada a diferentes áreas do conhecimento, a diversas organizações e setores, bem como associada a determinados produtos, processos, tecnologias.

De acordo com Müller Neto (2005), a inovação foi introduzida como um conceito fundamental para a explicação do desenvolvimento econômico, já no início do século XX, por Schumpeter, e, apesar de manter sua origem, o conceito evoluiu ao longo das últimas décadas, adquirindo um escopo bem mais amplo.

Segundo Schumpeter (1961), a inovação é definida como um processo caracterizado pela descontinuidade daquilo que está estabelecido. Podendo ser concebida através da introdução de um novo bem ou nova qualidade de um bem, de um novo método de produção, da abertura de um novo mercado, da conquista de uma nova fonte de matéria-prima ou, ainda, da criação de uma nova forma de organização.

De acordo com Kemp, Smith e Becher (2000), a inovação consiste em um fenômeno multifacetado, caracterizado por uma complexidade de inter-relações entre pessoas e instituições. Os autores enfatizam que a inovação envolve, de um lado, novas ideias e resoluções de problemas, podendo ser vista em termos de criatividade e esforço intelectual; e, de outro, recursos financeiros e materiais, usualmente em larga proporção e em condições incertas, com elevado risco.

Conforme exposto por Tidd, Bessant e Pavitt (2005), as inovações são decorrentes de processos organizacionais alinhados à estratégia organizacional e culminam com projetos que irão gerar vantagens competitivas, podendo ser proposto um modelo que explicite os pontoschave para a integração entre estratégia e inovação. Esses autores afirmam que o processo de inovação envolve as seguintes que etapas:

- prospectar o ambiente, tanto interno como externo, para identificar e processar sinais relevantes sobre ameaças e oportunidades relacionadas à mudança;

- decidir com base em uma visão estratégica a maneira como as empresas podem meIhor se desenvolver e a quais desses sinais devem responder;

- obter os recursos que possibilitem a resposta, seja criando algo novo, através de pesquisa e desenvolvimento, seja adquirindo algo externo, através de transferência de tecnologia; implementar o projeto (desenvolver a tecnologia e o mercado interno ou externo) para responder efetivamente.

Segundo Damanpour (1991), a inovação pode ser um novo produto ou serviço, uma nova tecnologia de processo de produção, um novo sistema administrativo, uma nova estrutura organizacional ou ainda um novo plano ou programa.

A inovação, quando relacionada especificamente aos produtos, aos serviços e à tecnologia dos processos de produção, é definida por Damanpour (1991) como inovação tecnológica. 
O Manual de Oslo (OCDE, 2005), define inovação tecnológica como a introdução de produtos ou processos tecnologicamente novos e melhorias significativas em produtos e processos existentes.

A inovação tecnológica, segundo Porter (1992), tem um papel importante no sucesso ou no fracasso de uma empresa, pois tem significantes implicações estratégicas para as empresas e pode influenciar a indústria como um todo.

Na visão de Daroit e Nascimento (2004), a inovação está comumente associada ao enfoque econômico, sendo tratada apenas como forma de obtenção de lucros extras pelas empresas, por meio de vantagens competitivas decorrentes da produção de novos produtos ou processos que agregam valor para o cliente. No entanto, esses autores enfatizam que é preciso uma maior compreensão por parte das organizações sobre o papel da inovação com relação a seus efeitos sobre a sociedade e o meio ambiente.

A partir desta maior preocupação, o conceito de desenvolvimento sustentável incorpora-se à gestão das organizações. O Relatório Brundtland, divulgado em 1987, pela Comissão Mundial sobre Meio Ambiente e Desenvolvimento (CMMAD), define desenvolvimento sustentável como o desenvolvimento capaz de suprir as necessidades da geração atual, sem comprometer a capacidade de atender às necessidades das futuras gerações (CMMAD, 1991).

Nesse sentido, o novo enfoque no âmbito organizacional, voltado para o desenvolvimento sustentável, busca unir o bem-estar econômico, a equidade social e a proteção ao meio ambiente.

As organizações sustentáveis são aquelas que desenvolvem suas práticas e estratégias gerenciais a fim de serem economicamente viáveis, mantendo-se competitivas no mercado, produzindo de maneira a não agredir o meio ambiente e contribuindo para o desenvolvimento social da região e do país onde atuam (ALMEIDA, 2002; LEAL, 2009).

Segundo Almeida (2007), o paradigma de desenvolvimento sustentável traz para as empresas dois grandes desafios: o primeiro consiste em gerar inovações necessárias à existência humana sustentável, disponibilizando soluções tecnológicas capazes de desempenhar múltiplas funções; o segundo está em vencer resistências da sociedade quanto aos novos produtos e serviços, particularmente sua descrença em relação a novas soluções frente às evidências da insustentabilidade dos sistemas produtivos e das atuais soluções tecnológicas incorporadas nos produtos e serviços que estão no mercado.

Nesse sentido, Hall e Vredenburg (2003) sustentam que a inovação tecnológica direcionada para o desenvolvimento sustentável opõe-se à forma convencional orientada para o mercado e requer tanto a incorporação das restrições oriundas das pressões sociais e ambientais como a incorporação de uma visão que leve em consideração também as futuras gerações, ou seja, a perspectiva de longo prazo.

A inovação orientada para a sustentabilidade é definida por Hansen, Grosse-Dunker e Reichwald (2009) como uma ferramenta que permite abranger tanto as questões de sustentabilidade como também conquistar novos segmentos de clientes e mercados, consistindo na inovação individualmente percebida por agregar valor positivo para o capital global da firma.

Dessa forma, o grande desafio das organizações está em incorporar a sustentabilidade no desenvolvimento de inovações, bem como nas suas estratégias de negócio, a fim de obter, cada vez mais, vantagens competitivas. 


\section{MÉTODO DO ESTUDO}

\subsection{Tipo de estudo}

Este estudo foi desenvolvido a partir de uma pesquisa bibliométrica, objetivando ampliar o conhecimento referente a Sustainable Innovation (Inovação Sustentável), na base de dados Web of Science no período de 2000 a 2010, e identificar quais tópicos em administração estudados junto a este tema estão sendo mais pesquisados e quais são mais relevantes.

Segundo Silva (2004), a bibliometria possui como objetivo analisar a atividade científica ou técnica através do estudo quantitativo das publicações. Complementando esta ideia, Rostaing (1997) afirma que o estudo bibliométrico consiste na aplicação dos métodos estatísticos ou matemáticos sobre o conjunto de referências bibliográficas. Para Macedo, Casa Nova e Almeida (2007), a bibliometria ajuda a conhecer o estágio em que uma pesquisa, em determinada área, encontra-se.

O estudo possui abordagem quantitativa, tendo em vista que procurou quantificar algumas variáveis referente à produção científica sobre Sustainable Innovation (Inovação Sustentável).

\subsection{Definição da amostra}

Os dados para realização desta pesquisa foram coletados através da base Web of Science do Institute for Scientific Information (ISI). Segundo Franceschet (2010), o ISI foi fundado por Eugene Garfield em 1960 e adquirido pela Thomson (hoje Thompson-Reuters) em 1992 e consiste em uma das maiores companhias do mundo da informação.

A Web of Science consiste em uma base multidisciplinar que indexa somente os periódicos mais citados em suas respectivas áreas. É também um índice de citações, informando, para cada artigo, os documentos por ele citados e os documentos que o citaram. Possui atualmente mais de 9.000 periódicos indexados (CAPES, 2010).

As referências de todos os itens indexados são extraídas, e a interface das referências citadas lista todas as citações de trabalhos às obras de um autor, independentemente dos itens citados serem indexados pela Web of Science ou não (BAR-ILAN, 2008).

Para tanto, a partir do mecanismo de busca da Web of Science, utilizando como palavrachave Sustainable Innovation e delimitando a busca para o período de 2000 a 2010 (11 anos), foram buscadas as publicações para análise.

\subsection{Modelo conceitual}

Para proceder à análise bibliométrica, o estudo buscou identificar as variáveis dispostas no quadro 1. 


\begin{tabular}{|c|c|}
\hline Características gerais das publicações & Número de citações de cada publicação \\
\hline$\checkmark \quad$ Total de publicações & $\checkmark \quad$ Índice h-b \\
\hline$\checkmark \quad$ Áreas temáticas & $\checkmark \quad$ Índice $\mathrm{m}$ \\
\hline$\checkmark \quad$ Tipos de documentos & \\
\hline$\checkmark \quad$ Ano das publicações & \\
\hline$\checkmark \quad$ Autores & \\
\hline$\checkmark \quad$ Título das fontes & \\
\hline$\checkmark \quad$ Instituições & \\
\hline$\checkmark \quad$ Agências financiadoras & \\
\hline$\checkmark \quad$ Países & \\
\hline$\checkmark \quad$ Idiomas & \\
\hline
\end{tabular}

Quadro 1 - Modelo conceitual para análise bibliométrica

O h-index (índice-h) foi proposto por Hirsch (2005) em sua pesquisa denominada An index to quantify an individual's scientific research output, como forma de caracterizar a produção científica de um pesquisador. Hirsch (2005) parte do princípio de que a quantificação do impacto e a relevância da produção científica individual são, muitas vezes, necessárias para a avaliação de pesquisadores e comparação de propósitos. Posteriormente, Banks (2006) propôs o índice $h$ - $b$, uma extensão do $h$-index, que é obtido através do número de citações de um tópico ou combinação em determinado período, listado em ordem decrescente de citações. $\mathrm{O}$ índice $h$ - $b$ é encontrado em publicações que tenham obtido um número de citações igual ou maior à sua posição no ranking. Banks (2006) também explica o cálculo do índice $m$, o qual é obtido através da divisão do índice h-b pelo período de anos que se deseja obter informações (n).

Para a análise dos índices $h$ - $b$ e $m$, foram utilizadas as definições de Banks (2006) evidenciadas no quadro 2.

\begin{tabular}{|l|ll|}
\hline \multicolumn{1}{|c|}{ Índice $\boldsymbol{m}$} & \multicolumn{1}{c|}{ Tópico/combinação } \\
\hline $0<\mathrm{m} \leq 0,5$ & $\checkmark$ & $\begin{array}{l}\text { Pode ser de interesse para pesquisadores em um campo específico de } \\
\text { pesquisa, o qual engloba uma comunidade pequena; }\end{array}$ \\
\hline $0,5<\mathrm{m} \leq 2$ & $\checkmark$ & $\begin{array}{l}\text { Provavelmente pode se tornar um hot topic como área de pesquisa, no qual a } \\
\text { comunidade é muito grande ou o tópico/combinação apresenta características } \\
\text { muito interessantes; }\end{array}$ \\
\hline $\mathrm{m} \geq 2$ & $\checkmark$ & $\begin{array}{l}\text { É considerado um hot topic, tópico exclusivo com alcance não apenas na sua } \\
\text { própria área de pesquisa e provavelmente tem e feitos de aplicação ou } \\
\text { características únicas. }\end{array}$ \\
\hline
\end{tabular}

Quadro 2 - Definições para classificação de hot topics

Fonte: Banks (2006).

A partir das definições de Banks (2006), neste estudo serão considerados hot topics as combinações com índice $\mathrm{m}=2$. 


\subsection{Etapas para a coleta dos dados}

A realização da pesquisa dividiu-se em quatro etapas. Inicialmente, digitou-se a palavra sustainable innovation como tópico no campo de pesquisa da Web of Science, delimitando-se o período de 2000 a 2010 (11 anos). Dessa forma, foram levantadas as informações: número total de publicações, áreas temáticas, tipo de documentos, ano das publicações, autores, título das fontes, instituições, agências financiadoras, países e idiomas.

$\mathrm{Na}$ segunda etapa, foram identificados os tópicos a serem relacionados com o tópico sustainable innovation. A partir de uma breve análise das publicações encontradas na primeira etapa, foram enumerados 20 tópicos, a serem combinados com o termo sustainable innovation.

Na terceira etapa, ocorreu a segunda busca ao sistema, combinando cada um dos tópicos ligados ao tema inovação sustentável com o termo sustainable innovation no período de 11 anos (2000 a 2010). Em seguida, na quarta etapa, realizou-se a classificação das publicações, e identificaram-se os hot topics, através do cálculo do índice $h-b$ e $m$.

A figura 1 evidencia as etapas da pesquisa.



Figura 1 - Etapas da pesquisa

Desse modo, de acordo com as etapas evidenciadas na figura 1, foi realizada a análise bibliométrica deste estudo, apresentada a seguir. 


\section{ANÁLISE E DISCUSSÃO DOS RESULTADOS}

Os resultados da pesquisa evidenciam as principais características da produção científica relacionada à expressão Sustainable Innovation. Primeiramente, pesquisou-se o termo na busca do Web of Science, no critério tópico e período de tempo de 2000 a 2010, sendo encontradas 1.022 publicações. Inicialmente, serão apresentadas as características gerais das publicações e, por fim, os hot topics relacionados ao tema.

\subsection{Características gerais das publicações sobre inovação sustentável no Web of Science}

A seguir, serão apresentadas as características gerais das publicações relacionadas ao tema, de acordo com as seguintes categorias: áreas temáticas, tipo de documentos, ano das publicações, autores, título das fontes, instituições, agências financiadoras, países e idiomas.

\subsection{1 Áreas temáticas das publicações}

A tabela 1 apresenta as 25 principais áreas temáticas relacionadas ao tema, de acordo com o número de publicações.

Tabela 1 - Áreas temáticas no estudo de inovação sustentável

\begin{tabular}{|c|c|}
\hline Área temática & № de publicações \\
\hline 1. Gestão (Management) & 201 \\
\hline 2. Ciências Ambientais (Environmental Sciences) & 168 \\
\hline 3. Negócios (Business) & 146 \\
\hline 4. Estudos Ambientais (Environmental Studies) & 115 \\
\hline 5. Engenharia Ambiental (Engineering, Environmental) & 88 \\
\hline 6. Planejamento e Desenvolvimento (Planning \& Development) & 88 \\
\hline 7. Economia (Economics) & 76 \\
\hline 8. Energia e Combustíveis (Energy \& Fuels) & 50 \\
\hline 9. Pesquisa Operacional e Ciência da Gestão (Operations Research \& Management Science) & 46 \\
\hline 10. Agricultura, Multidisciplinar (Agriculture, Multidisciplinary) & 45 \\
\hline 11. Engenharia Industrial (Engineering, Industrial) & 43 \\
\hline 12. Engenharia Multidisciplinar (Engineering, Multidisciplinary) & 43 \\
\hline 13. Geografia (Geography) & 42 \\
\hline 14. Ecologia (Ecology) & 35 \\
\hline 15. Ciências Multidisciplinares (Multidisciplinary Sciences) & 23 \\
\hline 16. Estudos Urbanos (Urban Studies) & 23 \\
\hline 17. Engenharia Química (Engineering, Chemical) & 20 \\
\hline 18. Sociologia (Sociology) & 20 \\
\hline 19. Engenharia Civil (Engineering, Civil) & 19 \\
\hline 20. Ciência da Informação e Biblioteconomia (Information Science \& Library Science) & 18 \\
\hline 21. Ciências da Saúde e Serviços (Health Care Sciences \& Services) & 16 \\
\hline 22. Metalurgia e Engenharia Metalúrgica (Metallurgy \& Metallurgical Engineering) & 16 \\
\hline 23. Biotecnologia e Microbiologia Aplicada (Biotechnology \& Applied Microbiology) & 15 \\
\hline 24. Construção e Tecnologia da Construção (Construction \& Building Technology) & 15 \\
\hline 25. Florestal (Forestry) & 15 \\
\hline
\end{tabular}

Em relação às áreas temáticas que abrangem a temática inovação sustentável, evidenciou-se que Gestão (Management), Ciências Ambientais (Environmental Sciences) e Negócio (Business) são aquelas que obtiveram um maior número de publicações. O fato de as áreas gestão e negócios ocuparem as primeiras posições indica que as publicações sobre inovação 
sustentável estão bastante relacionadas à área da Administração, representando um temática cada vez mais emergente no contexto empresarial.

\subsubsection{Tipos de documentos}

A tabela 2 apresenta os tipos de documentos referentes às publicações encontradas.

Tabela 2 - Classificação das publicações quanto ao tipo

\begin{tabular}{lcc}
\hline \multicolumn{1}{c}{ Tipo de publicação } & Frequência & Percentual \\
\hline Artigo & 775 & 75,83 \\
Paper em anais & 101 & 9,88 \\
Resenha & 94 & 9,20 \\
Material editorial & 39 & 3,82 \\
Resenha de livro & 7 & 0,68 \\
Resumo de reunião & 3 & 0,29 \\
Carta & 1 & 0,10 \\
Notícia & 1 & 0,10 \\
Reimpressão & 1 & 0,10 \\
\hline Total & $\mathbf{1 . 0 2 2}$ & $\mathbf{1 0 0}$ \\
\hline
\end{tabular}

A maioria das publicações encontradas são artigos, paper em anais e resenhas, evidenciando o caráter científico das mesmas.

\subsubsection{Publicações por ano}

No período compreendido entre 2000 e 2010, constatou-se que o número de publicações aumentou gradativamente ao longo dos 11 anos analisados. A figura 2 apresenta a quantidade de artigos publicados por ano relacionados ao tema inovação sustentável.

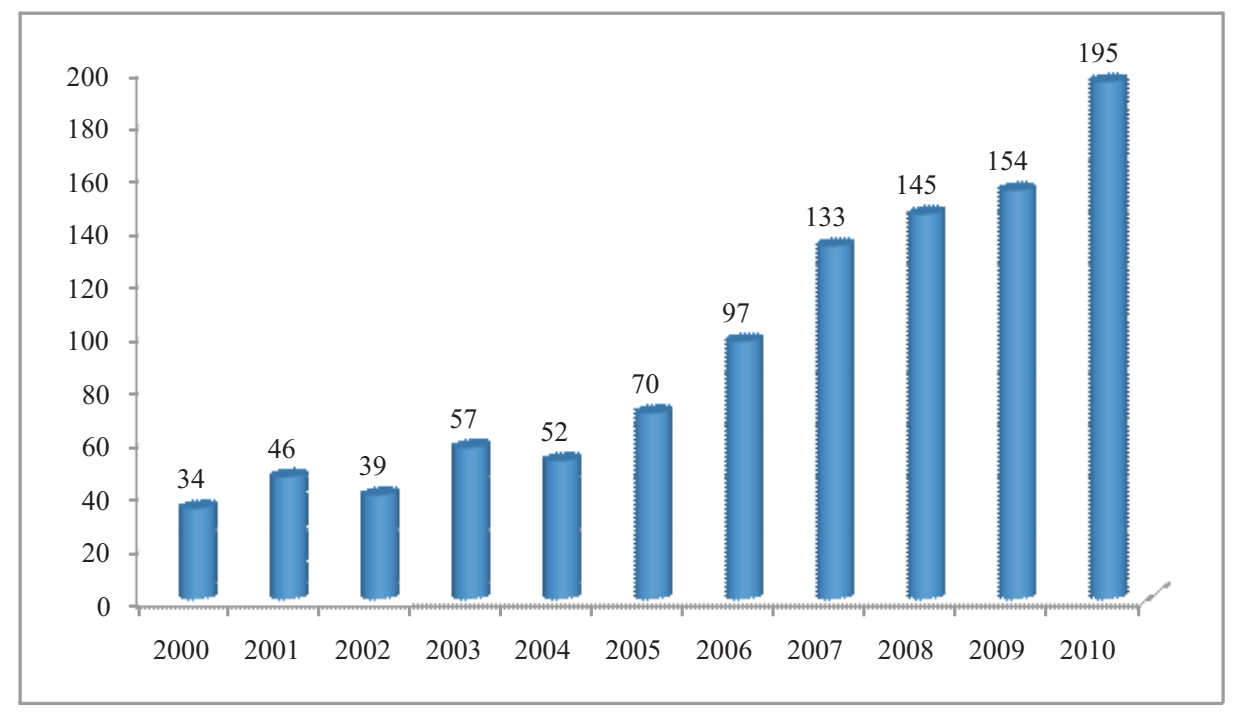

Figura 2 - Publicações por ano

Comparando o número de publicações do ano 2000 com 2010, evidencia-se que a quantidade de publicações relacionadas ao tema aumentou em quase seis vezes, demonstrando a emergência do tema. A partir dos resultados, observa-se que os estudos relacionados à 
inovação sustentável adquirem cada vez mais importância, tendo em vista que inovação e sustentabilidade estão diretamente relacionadas, já que o atual contexto competitivo estabelece que as empresas, ao inovar, necessitam considerar o impacto ao meio ambiente e à sociedade.

\subsubsection{Principais autores}

No que tange aos autores que mais publicaram sobre a temática inovação sustentável, desconsiderando as publicações não assinadas, foram listados os 24 autores, conforme a tabela 3 .

Tabela 3 - Quantidade de artigos publicados por autor

\begin{tabular}{|c|c|}
\hline Autor & Artigos publicados por autor \\
\hline Hekkert, M. P. & 8 \\
\hline Watanabe, C. & 7 \\
\hline Tukker, A. & 5 \\
\hline Carrillo-Hermosilla, J. & 4 \\
\hline Centi, G. & 4 \\
\hline Henard, D. H. & 4 \\
\hline Johannessen, J. A. & 4 \\
\hline Olsen, B. & 4 \\
\hline Smith, A. & 4 \\
\hline Vergragt, P. J. & 4 \\
\hline Vezzoli, C. & 4 \\
\hline Wiek, A. & 4 \\
\hline Alkemade, F. & 3 \\
\hline Belis-Bergouignan, M. C. & 3 \\
\hline Berkhout, F. & 3 \\
\hline Brown, J. & 3 \\
\hline Chia, E. & 3 \\
\hline
\end{tabular}

Evidenciou-se uma multiplicidade e diversidade quanto à autoria dos trabalhos, já que uma pequena parcela desses autores publicou um número elevado de artigos sobre a temática.

\subsubsection{Títulos das fontes}

A tabela 4 apresenta as 20 fontes que mais obtiveram publicações relacionadas à inovação sustentável. 
Tabela 4 - Principais fontes

\begin{tabular}{lc}
\hline \multicolumn{1}{c}{ Título da fonte } & № de artigos \\
\hline Journal of Cleaner Production & 26 \\
Energy Policy & 26 \\
Technological Forecasting and Social Change & 19 \\
International Journal of Technology Management & 16 \\
Ecological Economics & 15 \\
Technovation & 14 \\
Futures & 14 \\
Technology Analysis \& Strategic Management & 11 \\
Building Research and Information & 11 \\
Strategic Management Journal & 10 \\
Research-Technology Management & 9 \\
Journal of Business Ethics & 9 \\
Journal of Green Building & 9 \\
Research Policy & 9 \\
Agricultural Systems & 9 \\
Forestry Chronicle & 8 \\
International Journal of Engineering Education & 8 \\
European Planning Studies & 8 \\
Regional Studies & 7 \\
Renewable \& Sustainable Energy Reviews & 7 \\
\hline
\end{tabular}

A maioria das publicações referentes ao tema foi publicada nos periódicos: Journal of Cleaner Production, Energy Policy, Technological Forecasting and Social Change e International Journal of Technology Management.

\subsubsection{Principais instituições}

As 20 instituições que mais publicaram trabalhos relacionados à temática inovação sustentável estão evidenciadas na tabela 5.

Tabela 5 - Principais instituições

\begin{tabular}{|c|c|}
\hline Instituição & № de artigos \\
\hline Delft University of Technology & 20 \\
\hline University Utrecht & 18 \\
\hline Lund University & 11 \\
\hline University British Columbia & 11 \\
\hline Chinese Academy Of Sciences & 10 \\
\hline Harvard University & 10 \\
\hline VU University Amsterdam & 9 \\
\hline Wageningen University & 9 \\
\hline Open University & 8 \\
\hline University Michigan & 8 \\
\hline University Sussex & 8 \\
\hline University Washington & 8 \\
\hline Cornell University & 7 \\
\hline Cranfield University & 7 \\
\hline Free University Amsterdam & 7 \\
\hline Inland Northwest Research Alliance & 7 \\
\hline Tokyo Instute Technology & 7 \\
\hline University Leeds & 7 \\
\hline University Toronto & 7 \\
\hline Wageningen University and Research Centre & 7 \\
\hline
\end{tabular}


As instituições que mais se destacaram no que refere às publicações relacionadas à inovação sustentável foram: Delft University of Technology, University Utrecht, Lund University e University British Columbia.

\subsubsection{Principais agências financiadoras, países e idiomas}

Em relação às agências financiadoras de trabalhos que englobam inovação sustentável, enumeraram-se aquelas com maior número de publicações: European Commission (4), National Natural Science Foundation of China (3), Academy of Finland (2), Economic and Social Research Council - ESRC (2), National Science Foundation (2) e Robert Wood Johnson Foundation (2).

Quanto ao número de publicações por países, o Estados Unidos lidera o ranking dos que mais publicaram, com 236 estudos, seguido da Inglaterra (117), Holanda (106), Canadá (68), Alemanha (58), Austrália (56), Espanha (42), República Popular da China (40), França (39) e Suécia (33). Dessa forma, pode-se inferir que, nesses países, encontram-se as instituições que mais publicam e mais investem em pesquisa sobre inovação sustentável.

Corroborando os países que mais publicaram sobre a temática (Estados Unidos, Inglaterra, Holanda e Canadá), o idioma inglês se sobressai, com 961 publicações, totalizando 94\% do total. Em seguida, evidencia-se o espanhol, com 18 publicações, o alemão, com 15 estudos, e o francês, com 12 publicações. Os demais 16 trabalhos foram publicados em outros idiomas.

\subsection{O estudo da inovação sustentável e os hot topics}

Nesta etapa da pesquisa, foram investigadas as publicações sobre inovação sustentável e seus principais tópicos de estudo. Com base em uma análise prévia das publicações encontradas na Web of Science, foram selecionados 20 tópicos relacionados ao tema inovação sustentável.

Os tópicos selecionados foram: Business (Negócios), Strategy (Estratégia), Performance (Desempenho), Technology (Tecnologia), Development (Desenvolvimento), Growth (Crescimento), Energy (Energia), Water (Água), Eco-efficiency (Ecoeficiência), Management (Gestão), Supply Chain (Cadeia de suprimentos), Process (Processo), Product (Produto), Competitiveness (Competitividade), Knowledge (Conhecimento), Stakeholders (Partes interessadas), Globalization (Globalização), Industry (Indústria), Economy (Economia) e Planning (Planejamento).

A tabela 6 classifica os 20 tópicos selecionados conforme o número de publicações.

Posteriormente, foi realizada a combinação de cada tópico listado na tabela $6 \mathrm{com}$ a temática inovação sustentável, sendo calculado o total de publicações para cada combinação (tópico relacionado x sustainable innovation), o $h$-index e o coeficiente $m$ (Tabela 8). Os resultados foram listados conforme o total de publicações de cada combinação, porém é importante observar que vários tópicos que individualmente possuem um número expressivo de publicações (energy, growth, industry, knowledge, performance, process, product, strategy e water), quando pesquisados junto à temática inovação sustentável, possuem um número de publicações menos expressivo. 
Tabela 6 - Tópicos relacionados à inovação sustentável selecionados para a pesquisa

\begin{tabular}{|c|c|}
\hline Tópicos & Total de publicações \\
\hline $1^{\circ}$. Development (Desenvolvimento) & $>100.000$ \\
\hline $2^{\circ}$. Energy (Energia) & $>100.000$ \\
\hline $3^{\circ}$. Growth (Crescimento) & $>100.000$ \\
\hline $4^{\circ}$. Industry (Indústria) & $>100.000$ \\
\hline $5^{\circ} . \quad$ Knowledge (Conhecimento) & $>100.000$ \\
\hline 6. Management (Gestão) & $>100.000$ \\
\hline $7^{\circ}$. Performance (Desempenho) & $>100.000$ \\
\hline $8^{\circ}$. Process (Processo) & $>100.000$ \\
\hline $9^{\circ}$. Product (Produto) & $>100.000$ \\
\hline $10^{\circ}$. Strategy (Estratégia) & $>100.000$ \\
\hline 11‥ Technology (Tecnologia) & $>100.000$ \\
\hline $12^{\circ}$. Water (Água) & $>100.000$ \\
\hline 13‥ Planning (Planejamento) & 89.234 \\
\hline 14. Business (Negócios) & 57.159 \\
\hline 15․ Economy (Economia) & 49.954 \\
\hline 16. Globalization (Globalização) & 15.257 \\
\hline 170. Supply Chain (Cadeia de suprimentos) & 10.249 \\
\hline $18^{\circ}$. Stakeholders (Partes interessadas) & 12.287 \\
\hline 19. Competitiveness (Competitividade) & 7.254 \\
\hline 20․ Eco-efficiency (Ecoeficiência) & 355 \\
\hline
\end{tabular}

Tabela 7 - Hot topics no estudo de inovação sustentável

\begin{tabular}{|c|c|c|c|}
\hline Tópicos & $\begin{array}{c}\text { Total de } \\
\text { publicações }\end{array}$ & índice $h-b$ & índice $m$ \\
\hline 1ㅇ. Development (Desenvolvimento) & 648 & 27 & 2,45 \\
\hline 2‥ Management (Gestão) & 374 & 26 & 2,36 \\
\hline 3ㅇ. Technology (Tecnologia) & 329 & 24 & 2,18 \\
\hline 4‥ Performance (Desempenho) & 173 & 21 & 1,91 \\
\hline 5‥ Product (Produto) & 136 & 20 & 1,82 \\
\hline 6ㅇ. Strategy (Estratégia) & 159 & 19 & 1,73 \\
\hline 7o. Industry (Indústria) & 201 & 18 & 1,64 \\
\hline 8‥ Knowledge (Conhecimento) & 250 & 18 & 1,64 \\
\hline 9o. Process (Processo) & 239 & 18 & 1,64 \\
\hline 10‥ Growth (Crescimento) & 156 & 17 & 1,55 \\
\hline 11ㅇ․ Business (Negócios) & 174 & 17 & 1,55 \\
\hline 12‥ Energy (Energia) & 159 & 14 & 1,27 \\
\hline 13‥ Water (Água) & 76 & 12 & 1,09 \\
\hline 14ㅇ․ Economy (Economia) & 105 & 12 & 1,09 \\
\hline 15ㅇ․ Planning (Planejamento) & 74 & 11 & 1,00 \\
\hline 16ㅇ․ Competitiveness (Competitividade) & 62 & 9 & 0,82 \\
\hline 17o. Globalization (Globalização) & 22 & 6 & 0,55 \\
\hline $18^{\circ}$. Stakeholders (Partes interessadas) & 65 & 6 & 0,55 \\
\hline 19‥ Eco-efficiency (Ecoeficiência) & 11 & 6 & 0,55 \\
\hline 20‥ Supply Chain (Cadeia de suprimentos) & 19 & 4 & 0,36 \\
\hline
\end{tabular}


A partir do cálculo dos índices $h$ e $m$, é possível mensurar o desempenho dos tópicos/ combinações pesquisados, tendo por base o número de citações que estas tiveram (KELLY; JENNIONS, 2006). Orientando-se pelas considerações de Banks (2006), pode-se classificar como hot topcis, ou tópicos quentes, as combinações do tópico inovação sustentável com: Development (Desenvolvimento), Management (Gestão) e Technology (Tecnologia). As demais combinações, com exceção do tópico supply chain, por apresentarem $0,5<\mathrm{m}=2$, podem ser consideradas como hot topics emergentes como áreas de pesquisa. O tópico supply chain, por apresentar $\mathrm{m}=0,5$, pode ser de interesse para pesquisadores em um campo específico de pesquisa.

\section{CONSIDERAÇÕES FINAIS}

A análise das publicações sobre inovação sustentável na base de dados Web of Science, evidenciaram-se 1.022 publicações relacionadas ao tema, estando relacionadas principalmente às áreas temáticas: Gestão (Management), Ciências Ambientais (Environmental Sciences) e Negócio (Business).

Contatou-se que a maioria das publicações encontradas são artigos, sendo que, no período compreendido entre 2000 e 2010, a produção científica relacionada ao tema aumentou gradativamente ao longo dos 11 anos analisados. O Journal of Cleaner Production e a Delft University of Technology destacaram-se, com um maior número de publicações. Também constatou-se que o Estados Unidos lidera o ranking dos países que mais publicaram sobre a temática, e o idioma inglês é o predominante nas publicações. Evidenciaram-se como hot topcis, ou tópicos quentes, as combinações do tópico inovação sustentável com: Development (Desenvolvimento), Management (Gestão) e Technology (Tecnologia).

No decorrer do trabalho, foi possível verificar a utilidade de mecanismos de busca, como o Web of Science, para a realização de pesquisas acadêmicas, que servem de ferramenta para que a comunidade acadêmica tenha acesso às publicações, bem como busque informações a respeito da evolução de seus temas de interesse.

Estudos de natureza bibliométrica buscam ampliar a compreensão de um tema emergente, como a inovação sustentável. Além disso, servem também para demonstrar características relacionadas à produção científica, bem como autores e instituições que se destacam.

Os resultados desta pesquisa são relevantes para a construção do conhecimento científico sobre inovação sustentável, porém deve-se considerar como limitação do estudo o fato de ter sido realizado utilizando-se apenas uma base de dados específica. Por essa razão, sugere-se que estudos futuros desta natureza possuam uma amplitude maior, abrangendo, por exemplo, outros eventos acadêmicos nacionais e internacionais e também demais periódicos científicos. 


\section{REFERÊNCIAS BIBLIOGRÁFICAS}

ALMEIDA, F. O bom negócio da sustentabilidade. 1. ed. Rio de Janeiro: Nova Fronteira, 2002.

. Os desafios da sustentabilidade. Rio de Janeiro: Elsevier, 2007.

BANKS, M. G. An extension of the hirsch index: indexing scientific topics and compounds. 2006. Disponível em: <http:// www.arxiv.org/abs/physics/0604216>. Acesso em: 20 jul. 2010.

BAR-ILAN, J. Which h-index? A comparison of WoS, Scopus and Google Scholar. Scientometrics, v. 74, n. 2, p. 257-271, 2008.

BESSANT, J.; TIDD, J. Inovação e empreendedorismo. Porto Alegre: Bookman, 2009.

COORDENAÇÃO DE APERFEIÇOAMENTO DE PESSOAL DE NÍVEL SUPERIOR. Sobre Web of Science. Disponível em: <http:// buscador.periodicos.capes.gov.br.ez47.periodicos.capes.gov.br/ $\mathrm{V}$

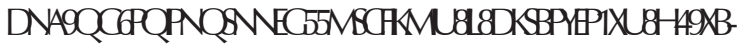

$\begin{array}{llllllll}3 & 4 & 0 & 7 & 2\end{array}$ ? $\mathrm{f}$ n $\mathrm{c}=\mathrm{f} \mathrm{i} \mathrm{n} \mathrm{d}-\mathrm{d}$ b info\&doc num $=000002653>$. Acesso em: 18 jan. 2011.

COMISSÃO MUNDIAL SOBRE MEIO AMBIENTE E DESENVOLVIMENTO. Nosso futuro comum. Rio de Janeiro: FGV, 1991.

DAMANPOUR, F. Organizational innovation: a meta-analysis of effects of determinants and moderators. Academy of Management Journal, v. 34, n. 3, p. 555-590, 1991.

DAROIT, D.; NASCIMENTO, L. F. Dimensões da inovação sob o paradigma do desenvolvimento sustentável. In: ENCONTRO NACIO-
NAL DA ANPAD, 28., 2004, Curitiba. Anais... Curitiba: Associação Nacional de Pós Graduação e Pesquisa em Administração, 2004. p. 116.

FRANCESCHET, M. A comparison of bibliometric indicators for computer science scholars and journals on Web of Science and Google Scholar. Scientometrics, v. 83, n. 1, p. 243-258, 2010.

HALL, J.; VREDENBURG, $\mathrm{H}$. The challenges of innovating for sustainable development. Mit Sloan Management Review, v. 45, n. 1, p. 61-68, 2003.

HANSEN, E.; GROSSE-DINKER, F.; REICHWALD, R. Sustainability innovation cube: a framework to evaluate sustainabilityoriented innovations. International Journal of Innovation Management, v. 13, n. 4, p. 683-713, 2009.

HIRSCH, J. E. An index to quantify an individual's scientific research output. Proceedings of the National Academy of Sciences of the United States of America, v. 102, n. 46, p. 16.569-16.572, 2005.

KELLY, C. D.; JENNIONS, M.D. The $h$-index and career assessment by numbers. Trends in Ecology \& Evolution, v. 21, p. 167-170, 2006.

KEMP, R.; SMITH, K.; BECHER, G. How should we study the relationship between environmental regulation and innovation? In: European Commission JRC-IPTS and Enterprise $D G$. The impact of $E U$ regulation on innovation of European Industry. Disponível em: <http://ftp.jrc.es/EURdoc/ eur19827en.pdf>. Acesso em: 20 jul. 2010.

LEAL, C. E. A era das organizações sustentáveis. Revista Eletrônica Novo Enfoque da Universidade Castelo Branco, Rio de Janeiro, v. 8, n. 8, jun. 2009. Disponível em: <http:/ /www.castelobranco.br/sistema/ novoenfoque>. Acesso em: 10 maio 2010. 
MACEDO, M. A. S.; CASA NOVA, S. P.; ALMEIDA, K. Mapeamento e análise bibliométrica da utilização da análise envoltória de dados (DEA) em estudos das áreas de contabilidade e administração. In: ENCONTRO DA ASSOCIAÇÃO NACIONAL DE PÓS GRADUAÇÃO E PESQUISA EM ADMINISTRAÇÃO, 31., 2007, Rio de Janeiro. Anais... Rio de Janeiro: ANPAD, 2007. p.1-16.

MÜLLER NETO, H. F. Inovação orientada para o mercado: um estudo das relações entre orientação para mercado, inovação e performance. 2005. 169 f. Tese (Doutorado em Administração) - Universidade Federal do Rio Grande do Sul, Porto Alegre, 2005.

OCDE. Manual de Oslo: diretrizes para Coleta e interpretação de dados sobre inovação. 3. ed. Rio de Janeiro: Finep, 2005.

PORTER, M. E. Vantagem competitiva: criando e sustentando um desempenho superior. Tradução de Elizabeth Maria de Pinho Braga. Rio de Janeiro: Campus, 1992.
SCHUMPETER, J. A. Capitalismo, socialismo e democracia. Rio de Janeiro: Fundo de Cultura, 1961.

SILVA, M. R. Análise bibliométrica da produção científica docente do programa de pós-graduação em educação especial/ UFSCar: 1998-2003. 168f. Dissertação (Mestrado em Educação Especial)-Universidade Federal de São Carlos, São Carlos, 2004.

ROSTAING, H. La bibliométrie et sés techniques. Toulouse: Sciences de la Société; n. 38; Marseille: Centre de Recherche Rétrospective de Marseille, 1996. p. 7-121.

TIDD, J.; BESSANT, J.; PAVITT, K. Managing innovation: integrating technological, market and organizational change. West Sussex: John Wiley \& Sons, 2005. 